\title{
Management of internal root resorption in primary mandibular right first molar: A case report with four-year follow-up
}

D) Prasad K Musale ${ }^{1} \bowtie$, (D) Sneha S Kothare ${ }^{2}$, (D) Abhinav l Talekar ${ }^{3}$

\section{Highlights}

The etiology and pathogenesis of internal inflammatory root resorption are partly understood and thus an attempt is made at the same.

${ }^{1}$ Chief paediatric dentist, Little One's Big Smiles, Laser and Microscopic Integrated Paediatric Dentistry, Pune, Maharashtra, India

2 Private practitioner, Mumbai,

Maharashtra, India

${ }^{3}$ Reader, Department of Pedodontics and Preventive Dentistry, M.A. Rangoonwala College of Dental Sciences and Research Centre, Pune, Maharashtra, India

\section{Correspondence:}

Little One's Big Smiles, Laser and Microscopic Integrated Paediatric Dentistry, Pune, Maharashtra, India E-mail address:

pedoprass@gmail.com
The importance of chemical debridement was emphasized.
An alternative to conventional methods of management of inflammatory internal root resorption was shown and suggested.

\section{Abstract}

Internal inflammatory root resorption in primary teeth is a well-known entity with unknown prevalence. The diagnosis and conservative treatment of such a lesion has perplexed practitioners for decades. The management of a nonperforating internal root resorption is long been debated by general dentists and paediatric dentists alike. This case presentation describes the case of a 4 year old boy with non-perforating internal root resorption in a primary molar treated successfully with endodontic procedure and monitored routinely for four years. The objective here was to suggest a minimally-invasive treatment alternative to conventional treatment practices.

Keywords: Primary Teeth; Pulpectomy; Root Resorption 


\section{INTRODUCTION}

Tooth resorption in the primary and permanent dentition has been studied extensively and the complex processes that lead to the loss of organic and inorganic components of tooth structure by clastic cells have been investigated. In recent times, Lindskog's classification has subdivided tooth resorptions into three broad groups: trauma induced tooth resorption; infection induced tooth resorption; and hyperplastic invasive tooth. ${ }^{1}$

Inflammatory resorption is defined as an internal or external pathologic loss of tooth structure and possibly bone, resulting in a defect, occurs as a result of microbial infection; characterized radiographically by radiolucent areas of the root. More specifically, internal root resorption is defined as a process initiated within the pulp space with loss of dentin and possible invasion of cementum. ${ }^{2}$ Thus, internal inflammatory root resorption (IRR) is a relatively rare type of tooth resorption that begins in the root canal and destroys surrounding dental hard tissues. It is a well-known entity with most dentists able to diagnose the phenomenon accurately. Available literature has described its exact clinical as well as radiographic appearance. However, its etiology, pathogenesis is only partially understood and its subsequent management has perplexed practitioners. Further, the occurrence and management of internal inflammatory root resorption in primary teeth is poorly understood and scant literature is available in this regard.

Trauma is the most common cause of IRR followed by pulpitis. Pulpotomy, cracked tooth, tooth transplantation, restorative procedures, invagination, orthodontic treatment and Herpes zoster viral infection are the other predisposing factors to this type of resorption. Untreated carious teeth, carious pulp involvement, pulpotomy, pulpectomy lead to higher occurrence of IRR in primary teeth. ${ }^{3}$ It is postulated that damage to the organic sheath, predentin and odontoblast cells covering mineralized dentine inside the root canal must occur to expose the mineralized tissue to pulpal cells with resorbing potential, thus resulting in loss of dentin and possible invasion of cementum. However, the exact initiating events leading to such denudation of root dentin are not fully understood. ${ }^{4}$

There is a general consensus that IRR is a rare phenomenon but its precise numbers are not known. ${ }^{5}$ Most of the available literature consists of case reports with emphasis on management of resorption. Haapsaalo and Endal ${ }^{4}$ theorized that the prevalence would be between 0.01 and 1\%.4 Gabor et al. ${ }^{5}$ reported that $50 \%$ of teeth with pulpitis and $77 \%$ of teeth with pulpal necrosis showed IRR while it was absent in healthy teeth. In primary molars, the prevalence is found to be $16.2 \%$ with greater prevalence in 3-7 year old children (19.4\%). ${ }^{3}$ Most of the lesions were in the middle third followed by apical third of the root while only one was in the coronal third. ${ }^{5}$

According to Wedenberg and Zetterqvist ${ }^{6}$, IRR in primary and permanent teeth is identical, both clinically and morphologically, except that the resorption is hastened in primary teeth. This difference could be attributed to the lesser number of clusters of epithelial cell rests of Mallassez in the periodontal ligament of primary teeth and lower expression of osteoprotegrin (OPG), factors usually associated with lower protection of primary teeth against root resorption. ${ }^{7}$

The development of root resorption requires two phases: injury to the protective tissues (mechanical or chemical) and then stimulation by infection or pressure. ${ }^{8}$

Bacteria-induced inflammation is typically accompanied by the production and release of potent stimulators of hard tissue resorption, such as osteoclast activating factor, prostaglandins, macrophage-chemotactic factor and bacterial 
lipopolysaccharides. This stimulation may be necessary for the progression of internal resorption. Cessation of IRR usually occurs once either total pulp necrosis occurs or once a pulpectomy is carried out because the dentinoclasts require a viable blood supply for them to survive and be active. ${ }^{4}$

IRR is usually asymptomatic and is often first diagnosed as an inadvertent finding on radiographic examination. Alternatively, it is found when the patient develops symptoms as a result of the progression of the disease process to cause acute apical periodontitis at some stage after the entire root canal system has become infected or if it perforates through the root communicating with the periodontal ligament (PDL). ${ }^{9}$ The normal radiographic presentation is an area of enlargement of the root canal or the pulp chamber. This enlargement can be symmetrical or eccentric but has sharp, smooth and clearly defined margins. The root canal is indistinguishable within the resorptive lesion and the size and location of the resorptive defect is variable depending on the extent of damage.

Although digital intraoral radiography provides an acceptable level of accuracy, digital subtraction radiography shows superior results. ${ }^{10}$ However, in recent times, CBCT is found to be superior to any form of two-dimensional radiography and may result in a review of the radiographic techniques used for assessing the type of resorption lesion present. This superior diagnostic accuracy also results in an increased likelihood of correct management of resorption lesions. ${ }^{11}$

An internal resorption lacuna, when evident radiographically, is a definite indication for endodontic treatment. Since the resorbing cells in this type of resorption are pulpal in origin, a pulpectomy would eliminate the granulation tissue and cut off the blood supply of the resorptive cells. ${ }^{8}$ Debridement and filling of the canal and the defect can be extremely challenging. Chemical debridement, which includes both irrigation and intracanal medication, has a major role to play in the elimination of the bacteria and the tissue present in the resorptive defect because this cannot be predictably achieved just with root canal instrumentation alone unless significant amounts of tooth structure are removed. However, such a mechanical approach will significantly weaken the tooth. Thus, the convention till date has been to extract the affected primary tooth and replace it with a suitable spacer maintainer. ${ }^{1}$ However, this case report discusses the management of a primary molar with non-perforating IRR and highlights the importance of chemical debridement with minimal mechanical preparation.

\section{CASE REPORT}

A 4-year old boy reported with a complain of severe pain and swelling associated with the primary mandibular right first molar since the previous night. His medical history was noncontributory. On clinical examination, a wellcircumscribed swelling was seen in the region of \#S on the buccal side. The gingiva overlying the region was reddish in color with reddish yellow color of the most prominent part. Clinically, the patient was diagnosed as a case acute dentoalveolar abscess of \#84. Radiographic evaluation of the tooth revealed a large, ovoid radiolucency extending from the cervical-third of the distal root to the middle-third that was confined within the dentinal walls. Diffuse interfurcal radiolucency was seen extending up to the apical region of the distal root not involving the developing tooth bud (Figure 1). Thus, the radiographic diagnosis was non-perforating type of internal root resorption with pulpal etiology. A decision to perform multi-visit pulpectomy was made based on the clinical and radiographic evaluation and informed consent was obtained for the same from the parents. 


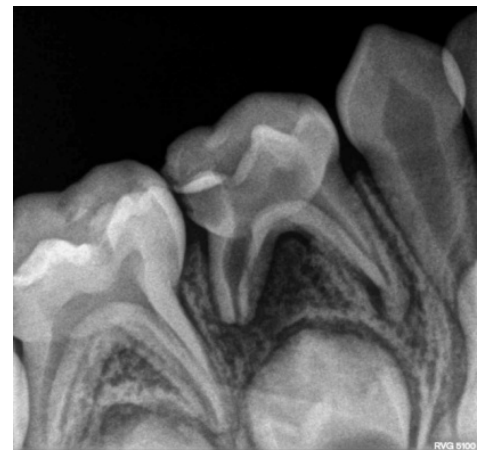

Figure 1. Pre-operative radiograph of primary mandibular right first molar showing non-perforating internal resorption in the distal root

As an emergency measure, caries excavation was done using \# 8 round bur (SS White, USA) and the pulp chamber was punched with the same for drainage of the abscess. Brisk bright- red bleeding was witnessed from the pulp chamber. A loose cotton pellet was placed in the coronal part for one week. Broad spectrum antibiotics were prescribed to the patient for five days.

After a week, resolution of the buccal abscess was seen on clinical examination with healthy gingival margin. After application of benzocaine $20 \% \mathrm{w} / \mathrm{v}$ topical anaesthetic gel (Ultracare, Ultradent Products Inc., USA) at the injection site, inferior alveolar nerve block was administered using 4\% Articaine with adrenaline (Septanest, Septodont, France) and the tooth was isolated using rubber dam. After isolation, the cotton pellet was removed and the root canal access was refined. A DG 16 endodontic explorer (Hu-Friedy, IL, USA) was used to locate the two mesial and one distal canals. The pulp was extirpated and a glide path was created using a \#10 K-file (Dentsply/Maillefer, USA). The root canals were minimally prepared using \#S2 Universal Protaper Gold (Dentsply/Maillefer, USA) rotary files. Copious canal irrigation and disinfection was performed with $1 \%$ sodium hypochlorite solution at $300 \mathrm{rpm}$ with constant low torque setting $(1.5 \mathrm{~N} . \mathrm{cm})$ as per the manufacturer's instructions. After the chemomechanical preparation, all canals were dried using absorbent paper points and a non- setting high $\mathrm{pH}$ calcium hydroxide intracanal medicament (Ultracal XS, Ultradent Products Inc., USA) was placed within the canals and the tooth was temporized (Figure 2).

The patient was recalled after 7 days. In the recall appointment, the tooth was again isolated under local anaesthesia and the root canals were flushed with sterile saline to remove any residual calcium hydroxide. After irrigation, the canals were inspected and dried using absorbent paper points. After drying, obturation was done with calcium hydroxide and iodoform-based paste (DiaPex Plus, DaiDent, Korea). Following obturation, the tooth was restored with IRM (Caulk-Dentsply, USA) and a preformed stainless steel crown (3M ESPE Dental Products, USA) cemented as a definitive restoration. Some amount of the obturating material was extruded beyond the apex (Figure 3). Oral hygiene instructions were given to the patient and a regular follow-up was recommended.

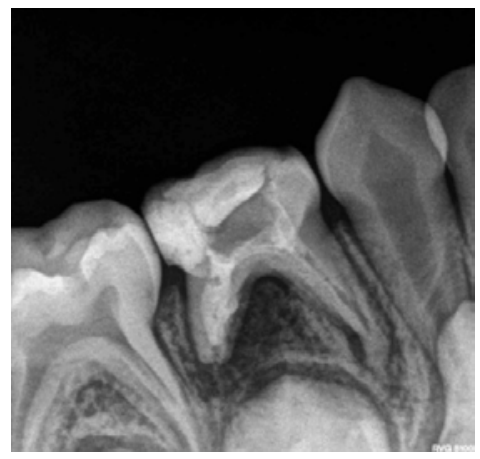

Figure 2. Non-setting high $\mathrm{pH}$ calcium hydroxide intracanal medicament placed within the canals and temporization done

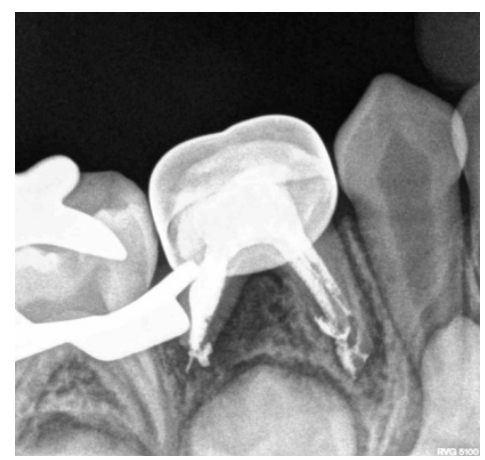

Figure 3. Post-operative radiograph showing slight extrusion of DiaPex Plus beyond the apex and definitive stainless steel crown 
One week post-operatively, the patient was asymptomatic and clinically, the soft tissues had healed with healthy surrounding tissues. In the 6month follow-up, radiographic evaluation showed loss of extruded obturation material, progressive resolution of the interfurcal lesion and stabilization of the resorption defect (Figure 4). Continuous margin of the developing tooth bud is seen. The one- (Figure 5) and two-year follow ups, showed healthy resolution of the lesion with

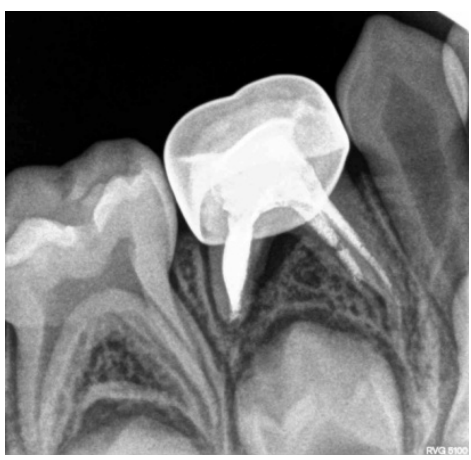

Figure 4. Six-month follow-up radiograph showing progressive resolution of the interfurcal lesion and stabilization of the resorption defect

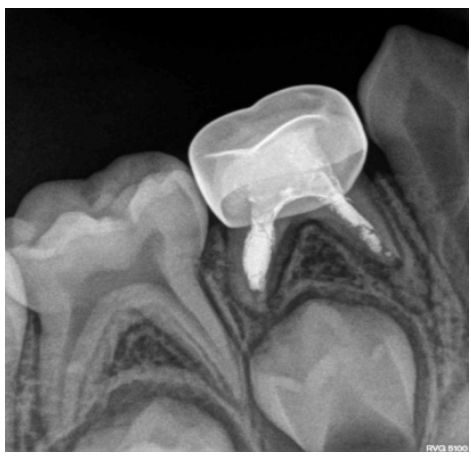

Figure 6. Two-year follow-up showing healthy resolution of the lesion with normal physiologic root resorption normal physiologic root resorption (Figure 6). After three years, it was noticed that the rate of resorption of the mesial root is greater than the distal root (Figure 7). The four- year follow-up shows considerable physiologic mesial root resorption while the rate of resorption in distal is slower. The continued root formation of the succedaneous premolar corresponds with the dental age of the patient (Figure 8).

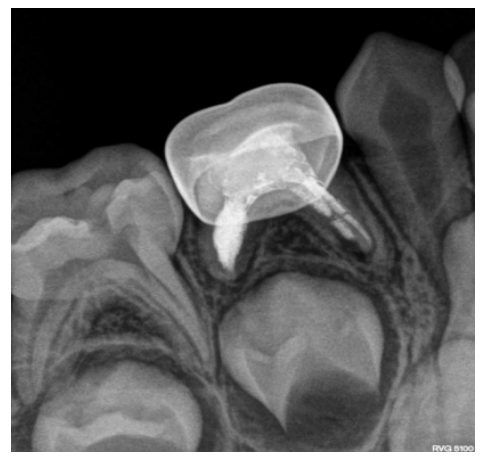

Figure 5. One-year follow-up showing complete resolution of the interfurcal lesion

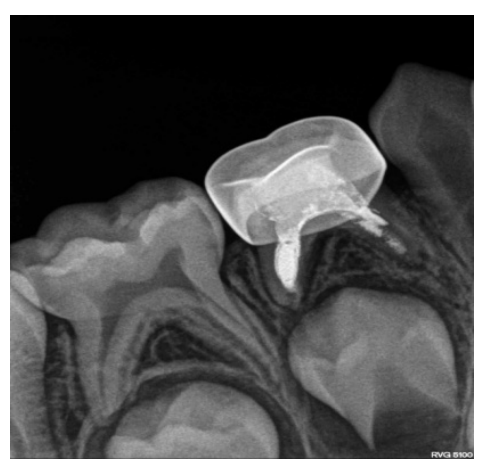

Figure 7. Three-year follow-up shows greater root resorption in the mesial roots than the distal root

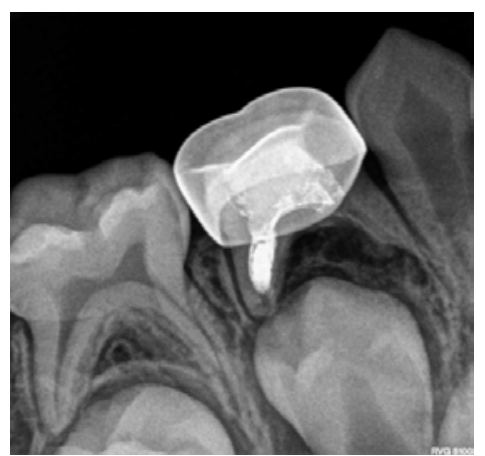

Figure 8. Four-year follow-up shows considerable physiologic mesial root resorption and normal root formation of succedaneous premolar 


\section{DISCUSSION}

Inflammatory internal root resorption, once thought to have a poor prognosis and thus indicated for extraction, has a good prognosis after treatment if the lesion is small and not perforated.

Instrumentation in internal root resorption is kept minimal to prevent further weakening of the already thinned dentinal walls. Further, due to the ovoid shape of the resorption lesion, excessive instrumentation is of no consequence in debridement of the lesion and thus the emphasis has been on chemical debridement with minimal mechanical instrumentation.

In the present case, brisk bleeding, signifying active resorption process or vital nature of the pulp, was controlled with $1 \%$ sodium hypochlorite irrigation ${ }^{4}$ in the second appointment. This irrigation not only controlled the bleeding but also necrotized the vital tissue. In the following appointment, repeat sodium hypochlorite irrigation helped dissolve the residual necrotic tissue and disinfect the canals prior to obturation.

The inter-appointment calcium hydroxide medicament has two important functions as to control bleeding and necrotize residual pulp tissue to make it more soluble to sodium hypochlorite. ${ }^{3}$ In the present case, the high $\mathrm{pH}$ non-setting calcium hydroxide intracanal medicament was left in place for one week ${ }^{12}$ to facilitate removal of the residual tissue at the next appointment by irrigation and instrumentation.

The treatment of choice for IRR in primary teeth has been extraction followed by replacement with suitable space maintainer. ${ }^{13}$ However, several less invasive alternatives are now suggested. In this case report, the obturating material used was Diapex. Similar endodontic management was done in case of multiple idiopathic internal resorption lesions using Vitapex. ${ }^{14}$ Choi et al. ${ }^{15}$ reported stabilization of IRR following pulp therapy in a child with severe atopic dermatitis.
Mineral trioxide aggregate (MTA) has also been suggested as an alternative medicament for management of IRR in primary molars. ${ }^{16}$

\section{CONCLUSIONS}

Inflammatory internal root resorption is a treatable entity with a good prognosis. However, further research, in this regard, is necessary with longer follow-up periods and greater population under study.

\section{REFERENCES}

1. Wedenberg C, Lindskog S. Experimental internal resorption in monkey teeth. Dent Traumatol 1985;1:221-227

2. American Academy of Endodontists. Glossary of Endodontic Terms [Internet] 2016 [cited 2021 Feb 13]. Avaliable from: www.aae.org/glossary

3. Vieira-Andrade RG, Drumond CL, Alves LPA, Marques LS, Ramos-Jorge ML. Inflammatory root resorption in primary molars: prevalence and associated factors. Braz Oral Res 2012;26:335-340

4. Haapasalo M, Endal U. Internal inflammatory root resorption: the unknown resorption of the tooth. Endod Top 2006;14:60-79

5. Gabor C, Tam E, Shen Y, Haapasalo M. Prevalence of internal inflammatory root resorption. J Endod 2012;38:24-27

6. Wedenberg C, Zetterqvist L. Internal resorption in human teeth-a histological, scanning electron microscopic, and enzyme histochemical study. J Endod 1987;13:255-259

7. Cordeiro MMR, Santos BZ, Reyes-Carmona JF, Figueiredo CP. Primary teeth show less protecting factors against root resorption. Int J Paediatr Dent 2011;21:361-368

8. Fuss Z, Tsesis I, Lin S. Root resorption-diagnosis, classification and treatment choices based on stimulation factors. Dent Traumatol 2003;19:175-182

9. Tronstad L. Root resorption- etiology, terminology and clinical manifestations. Endod Dent Traumatol 1988;4:241-52

10 .Stephanopoulos G, Mikrogeorgis G, Lyroudia $\mathrm{K}$. Assessment of simulated internal resorption 
cavities using digital and digital subtraction radiography: A comparative study. Dent Traumatol 2011;27:344-349

11. Patel S, Dawood A, Wilson R, Horner K, Mannocci $F$. The detection and management of root resorption lesions using intraoral radiography and cone beam computed tomography- an in vivo investigation. Int Endod J 2009;42:831-838

12. Rodd HD, Waterhouse PJ, Fuks AB, Fayle SA, Moffat MA. UK National Clinical Guidelines in Paediatric Dentistry: stainless steel preformed crowns for primary molars. Int J Paediatr Dent 2008; 18:20-28

13. Smith NL, Seale NS, Nunn ME. Ferric sulfate pulpotomy in primary molars: a retrospective study. Pediatr Dent 2000;22:192-199

14. Matsumoto-Nakano M, Kamakura N, Miyamoto E, Okawa R, Inagaki S, Fukuda Y, Ooshima T. Multiple sites of idiopathic internal resorption in primary dentition: A case report. Pediatr Dent J 2009;19:150-153

15. Choi EJ, Ahn BD, Lee JI, Kim JW. Multiple internal resorptions in deciduous teeth: a case report. J Oral Pathol Med 2007;36:250-251

16. Sari S, Sönmez D. Internal resorption treated with Mineral Trioxide Aggregate in a primary molar tooth: 18-month follow-up. J Endod 2006;32:69-71

\section{How to cite this article:}

Prasad K Musale, Sneha S Kothare, Abhinav L Talekar. Management of internal root resorption in primary mandibular right first molar: A case report with four-year follow-up. Contemp Pediatr Dent 2021:2(1):57-63

\section{Declarations}

Acknowledgements: Not applicable.

Conflict of Interest Statement: The authors disclose no potential conflicts of interest.

Ethics Statement: Procedure was explained to the parents and written consent was obtained for the treatment and publication of the case report.

Informed Consent: Written consent was taken for participation of parents and child in the study.

Author contributions: Conception and design: All Authors; Acquisition of data: PKM; Interpretation of data: PKM, SSK; Drafting article: All Authors; Revision artice: All Authors; Final approval: All Authors

Funding: This work is not finantiated.

Data Availability: The data used to support the findings of this study can be made available upon request to the corresponding author.

Peer-review: Externally double-blinded peer-reviewed. 